ARTICLE

\title{
Structural ensemble of a glutamate transporter homologue in lipid nanodisc environment
}

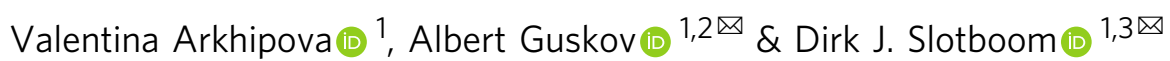

Glutamate transporters are cation-coupled secondary active membrane transporters that clear the neurotransmitter L-glutamate from the synaptic cleft. These transporters are homotrimers, with each protomer functioning independently by an elevator-type mechanism, in which a mobile transport domain alternates between inward- and outward-oriented states. Using single-particle cryo-EM we have determined five structures of the glutamate transporter homologue $\mathrm{Glt}_{T \mathrm{k}}$, a $\mathrm{Na}^{+}$- L-aspartate symporter, embedded in lipid nanodiscs. Dependent on the substrate concentrations used, the protomers of the trimer adopt a variety of asymmetrical conformations, consistent with the independent movement. Six of the 15 resolved protomers are in a hitherto elusive state of the transport cycle in which the inwardfacing transporters are loaded with $\mathrm{Na}^{+}$ions. These structures explain how substrate-leakage is prevented - a strict requirement for coupled transport. The belt protein of the lipid nanodiscs bends around the inward oriented protomers, suggesting that membrane deformations occur during transport.

\footnotetext{
${ }^{1}$ Groningen Biomolecular Sciences and Biotechnology Institute (GBB), University of Groningen, Nijenborgh 4, 9747AG Groningen, The Netherlands.

${ }^{2}$ Moscow Institute of Physics and Technology, Dolgoprudny, Russia. ${ }^{3}$ Zernike Institute for Advanced Materials, University of Groningen, Nijenborgh 4 ,

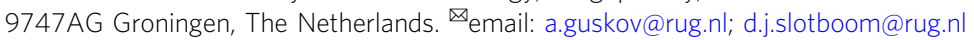


n humans, glutamate transporters (also known as excitatory amino acid transporters, EAATs) take up the neurotransmitter L-glutamate from the synaptic environment, which is necessary to sustain efficient neuronal communication and prevent neurotoxicity ${ }^{1-3}$. EAATs couple uptake of one substrate molecule to the transport of three sodium ions and one proton and counter-transport of one potassium ion ${ }^{4-6}$. Prokaryotic glutamate transporters homologs share high structural similarity with EAATs and neutral amino acid transporters ASCTs, which all belong to the solute carrier family 1 (SLC1A $)^{7}$. Structural studies of the archaeal homologs Glt $\mathrm{Ph}_{\mathrm{Ph}}$ from Pyrococcus horikoshii and $\mathrm{Glt}_{\mathrm{Tk}}$ from Thermococcus kodakarensis have provided the structural basis for understanding of the transport mechanism ${ }^{8-13}$. Both Glt $t_{\mathrm{Ph}}$ and $\mathrm{Glt}_{\mathrm{Tk}}$ couple uptake of one aspartate molecule to symport of three sodium ions ${ }^{12,14}$.

Glutamate transporters and their archaeal homologs are homotrimers, in which each protomer consists of a rigid scaffold domain involved in oligomerization and anchoring of the protein in the membrane, and a mobile transport domain that binds the substrate and cations and transports its cargo in an elevator-like motion across the membrane ${ }^{10}$. During movement of the transport domain, the substrate binding site is occluded from the solvent by two pseudo-symmetrical helical hairpins HP1 and HP2. The latter hairpin was shown to work as both an extracellular $9,15,16$ and an intracellular ${ }^{17}$ gate.

Mutagenesis studies ${ }^{18-20}$, single molecule fluorescence resonance energy transfer (smFRET) ${ }^{21,22}$, high-speed atomic force microscopy studies (HS-AFM) ${ }^{23}$, and molecular dynamics (MD) simulations ${ }^{24,25}$ strongly indicate that the transport domains of the three protomers move independently, hence should frequently visit asymmetric states during turnover conditions. However, extensive structural studies of glutamate transporters have revealed almost exclusively symmetrical arrangements of transport domains either in outward or inward states $9,10,12,17,26-28$. The only asymmetric state observed to date is in the crystal structure of a Glt $_{\mathrm{Ph}}$ mutant $^{29}$. The discrepancy between the structural information on these trimeric proteins and the data from dynamics studies may originate from the detergent-solubilized state, which was used for determination of all reported structures, and that is a very different environment compared to the native membrane. Reconstitution of proteins into lipid nanodiscs formed by scaffold proteins allows to better mimic the lipid bilayer environment and may help to avoid some of the possible detergent artefacts ${ }^{30}$.

Here we report cryo-EM structures of the glutamate transporter homolog $\mathrm{Glt}_{\mathrm{Tk}}$ in nanodiscs, revealing a variety of asymmetric arrangements of the transport domains. In addition, previously undetected conformations of the individual protomers provide structural insight in the elevator mechanism.

\section{Results}

Cryo-EM structures of Glt $_{\mathrm{Tk}}$ in nanodiscs. Purified $\mathrm{Glt}_{\mathrm{Tk}}$ was reconstituted into nanodiscs using the MSP2N2 scaffold protein ${ }^{31}$ and a mixture of E.coli polar lipids and egg PC (3:1 (w/w)), a lipid composition that supports robust transport activity of the protein in proteoliposomes ${ }^{11,32}$. $\mathrm{Glt}_{\mathrm{Tk}^{-}}$-nanodiscs were concentrated to 4.5-9.0 $\mu \mathrm{M}$, and supplemented with $300 \mathrm{mM} \mathrm{Na}{ }^{+}$. To these preparations we added either nothing $\left(\mathrm{Na}^{+}\right.$-only condition), or different concentrations of L-aspartate, or the competitive inhibitor DL-threo-beta-benzyloxyaspartate (TBOA inhibited). The preparations were analyzed by single particle cryo-electron microscopy with the aim to obtain structural insight in the conformational ensemble under turnover and stalled conditions (See Table 1 and Supplementary Fig. 1 for the cryo-EM workflows). We solved five structures of $\mathrm{Glt}_{\mathrm{Tk}}$ (with resolutions of 3.2-3.5 $\AA$, Supplementary Fig. 2), each with the protomers in a different trimeric arrangement (Fig. 1). In the collective set of structures, the 15 individual protomers adopted four different conformations (Fig. 1), which are characterized by their position relative to the scaffold domain (inward, outward, or intermediateoutward), the accessibility of the aspartate binding site (open or occluded), and the presence of substrates (apo, $\mathrm{Na}^{+}$-only, holo (Asp), holo (TBOA)). With one exception (when the $\mathrm{Glt}_{\mathrm{Tk}}$ binding sites were saturated with L-aspartate), the arrangements of the transport domains in the trimer are non-symmetrical.

Aspartate-free conditions. Cryo-EM analysis of $\mathrm{Glt}_{\mathrm{Tk}}$ in the presence of $300 \mathrm{mM} \mathrm{Na}^{+}$, but in the absence of the substrate Laspartate showed an asymmetric trimer with two transport domains in an inward position, and the third one in an intermediate-outward position. The latter transport domain has an occluded binding site, with the HP2 gate closed (distance between the tips of HP1 and HP2 $4.4 \AA$, Supplementary Table 1). We infer that this protomer is in the apo state, because the conformation of the transport domain is identical to that of the transport domains in the apo-crystal structure of $\mathrm{Glt}_{\mathrm{Tk}}(\mathrm{PDB}$ 5DWY, rmsd $0.534 \AA)^{12}$. In the apo-state the binding sites of sodium ions are deformed, and the binding site for aspartate is non-existent because the conformation of the chain of Arg401 is incompatible with aspartate binding (Fig. 2a). Despite the similarity in conformation of the apo-transport domains in the cryoEM and crystal structures, the position of the transport domain relative to the scaffold domain is different. In the cryo-EM structure, the transport domain is in an intermediate-outward position, whereas in the crystal structure (PDB $5 \mathrm{DWY})^{12}$ it is fully outward. The position of the transport domain is roughly similar to that of an intermediate-outward domain observed in a crystal structure of Glt $_{\mathrm{Ph}}$ (PDB 3V8G, chain $\mathrm{C}^{29}$; rmsd 1.261 $\AA$, Supplementary Fig. S3a), but whereas the $\mathrm{Glt}_{\mathrm{Ph}}$ protomer was in a holo state $\left(\mathrm{Na}^{+}\right.$-bound and Asp-bound), the Glt $\mathrm{Tk}_{\mathrm{Tk}}$ protomer is in the apo state.

In the two inward-oriented transport domains the gate formed by HP2 is open (distance between the tips of HP1 and HP2 9.3 $\AA$, Supplementary Table 1), and the empty binding site for aspartate (Fig. 2c) is accessible to the aqueous solution. The conformation of the protomers strongly suggests that the inwardopen protomers are in a $\mathrm{Na}^{+}$-bound state. This interpretation is based on the observation that two of the three binding sites for $\mathrm{Na}^{+}$ions ( $\mathrm{Na} 1$ and $\mathrm{Na} 3$ ), which are deformed in the apo protein $^{12}$, are reshaped in the cryo-EM structure, consistent with bound $\mathrm{Na}^{+}$ions. The presence of cryo-EM density in these sites further supports the interpretation that sodium ions are indeed bound, although the densities do not allow unequivocal assignment at the obtained resolution of $3.2 \AA$ (Supplementary Fig. 4a). The Na2 site is not formed properly, because the open HP2 gate is incompatible with the Na2 site geometry (Supplementary Fig. 4b). A prominent indicator for the $\mathrm{Na}^{+}$-bound geometry at sites $\mathrm{Na} 1$ and $\mathrm{Na} 3$ is the conformation of the central unwound region of transmembrane helix 7 (TM7), around Met314. The sidechain of Met314 points away from the binding site in the apo state, as first shown in a crystal structure of $\mathrm{Glt}_{\mathrm{Tk}}{ }^{12}$, and later also observed for Glt $_{\mathrm{Ph}^{3}}{ }^{33}$ (see also Fig. 2a), but is rotated over a distance of $9.7 \AA$ in the bound state.

$\mathrm{Na}^{+}$binding was shown previously to lead allosterically to formation of a high-affinity aspartate-binding site ${ }^{11,12,34,35}$. In the cryo-EM structure of the inward-oriented protomers, the side chain of Arg401, which adopts a conformation incompatible with aspartate binding in the apo-state, indeed has taken the position required for high-affinity L-aspartate binding (Fig. 2a). While an L-aspartate binding site is present in these protomers, it is unoccupied, consistent with a lack of cryo-EM 
Table 1 Cryo-EM data collection, refinement and validation.

\begin{tabular}{|c|c|c|c|c|c|}
\hline Voltage (kV) & 200 & 200 & 200 & 200 & 200 \\
\hline Defocus range $(\mu \mathrm{m})$ & -0.5 to -2.0 & -0.5 to -2.0 & -0.5 to -2.0 & -0.5 to -2.0 & -0.5 to -2.0 \\
\hline Pixel size $(\AA)$ & 1.012 & 1.012 & 1.012 & 1.012 & 1.012 \\
\hline Symmetry imposed & $\mathrm{C} 1$ & $\mathrm{C} 1$ & $\mathrm{C} 1$ & $\mathrm{C} 3$ & $\mathrm{C} 1$ \\
\hline Initial dataset (\# of particles) & $1,193,046$ & 348,173 & 348,173 & 444,955 & 578,728 \\
\hline \multicolumn{6}{|l|}{ Refinement } \\
\hline Initial model used & PDB 5E9S & PDB 5E9S & PDB 5E9S & PDB 5E9S & PDB 5E9S \\
\hline Map-sharpening $B$ factor $\left(\AA^{2}\right)$ & 84.8 & 101.7 & 81.0 & 129.3 & 135.9 \\
\hline \multicolumn{6}{|c|}{ Model composition } \\
\hline Nonhydrogen atoms & 9564 & 9555 & 9552 & 9572 & 9547 \\
\hline Protein residues & 1278 & 1281 & 1280 & 1281 & 1276 \\
\hline \multicolumn{6}{|l|}{ R.m.s. deviations } \\
\hline Bond lengths $(\AA)$ & 0.008 & 0.006 & 0.007 & 0.003 & 0.004 \\
\hline Bond angles $\left({ }^{\circ}\right)$ & 0.747 & 0.684 & 0.708 & 0.600 & 0.610 \\
\hline \multicolumn{6}{|l|}{ Validation } \\
\hline MolProbity score & 1.95 & 1.92 & 1.95 & 1.79 & 1.80 \\
\hline Clash score & 10.98 & 9.83 & 10.65 & 10.27 & 10.91 \\
\hline Poor rotamers (\%) & 0.00 & 0.00 & 0.00 & 0.00 & 0.10 \\
\hline \multicolumn{6}{|l|}{ Ramachandran plot (\%) } \\
\hline Favored & 94.26 & 94.03 & 93.87 & 96.23 & 96.38 \\
\hline Allowed & 5.74 & 5.97 & 6.13 & 3.54 & 3.62 \\
\hline Outliers & 0.00 & 0.00 & 0.00 & 0.24 & 0.00 \\
\hline Model to map fit CC & 0.83 & 0.8 & 0.84 & 0.77 & 0.78 \\
\hline
\end{tabular}

density (Fig. 2c). The protomers are thus in a hitherto elusive $\mathrm{Na}^{+}$-only state.

The fact that we observe one protomer in the apo state and two in $\mathrm{Na}^{+}$-bound states, indicates that we did not manage to saturate all protomers with $\mathrm{Na}^{+}$using a concentration of $300 \mathrm{mM} \mathrm{Na}{ }^{+}$. This observation is consistent with reported $K_{\mathrm{d}}$ values for sodium binding to Glt $t_{\mathrm{Ph}}(100-140 \mathrm{mM})^{36}$. Because of the sub-saturating $\mathrm{Na}^{+}$concentration, $\mathrm{Glt}_{\mathrm{Tk}}$ trimers with different ratios of apo and $\mathrm{Na}^{+}$-bound protomers were also expected in the dataset. Analysis of particles discarded during heterogeneous refinement for this dataset indeed revealed the presence of another asymmetric species with one inward-oriented and two outward-oriented transport domains, but this structure could be refined only up to $4.8 \AA$ resolution. Possibly, the collection and analysis of more particles would allow the determination of this structure at higher resolution, as well as the detection of $\mathrm{Glt}_{\mathrm{Tk}}$ in other states described by the binomial distribution of protomers over the apo and $\mathrm{Na}^{+}$-bound states, similar to what we will describe below for the substrate-unsaturated conditions.

Fully loaded state. At the concentration of $300 \mathrm{mM} \mathrm{Na}^{+}$used in the experiments presented here, the apparent $\mathrm{K}_{\mathrm{d}}$ for $\mathrm{L}$-aspartate is $\sim 120 \mathrm{nM}^{32}$, and therefore addition of $50 \mu \mathrm{M}$-aspartate to $5.6 \mu \mathrm{M}$ nanodiscs is expected to lead to substrate saturation. The cryoEM structure of $\mathrm{Glt}_{\mathrm{Tk}}$ in the holo (Asp) state is symmetrical with all three transport domains in an intermediate-outward position (Figs. 1, 3a). This position is similar to the ones found in a protomer of $\mathrm{Glt}_{\mathrm{Ph}}$ (PDB 3V8G, chain $\mathrm{C}^{29}$; rmsd $0.965 \AA$, Supplementary Fig. $3 \mathrm{~b}$ ) and in the apo state described above, showing that the intermediate-outward orientation is visited by both the holo and apo transporters (Fig. 3b). In addition, the HP2 gates are closed in both cases, consistent with the ability to make elevatortype movements (Supplementary Table 1). Despite these similarities, there are also conspicuous differences. In the cryo-EM map of the holo (Asp) state, density for the amino acid substrate in the binding site is observed (Fig. 2b). Although the resolution is not high enough to unambiguously assign the density to aspartate, the positions of the binding residues are virtually the same as found in the crystal structure of $\mathrm{Glt}_{\mathrm{Tk}}$ with L-aspartate bound (PDB $5 \mathrm{E} 9 \mathrm{~S})^{12}$. The conformation of residues involved in binding of the three $\mathrm{Na}^{+}$-ions also differs between the apo and holo (Asp) states, with the correct binding site geometries only adopted in the latter. Finally, the C-terminal half of TM7, the helices of HP2 and the $\mathrm{N}$-terminal half of TM8 in the holo (Asp) state are displaced away from the center of the trimer by $\sim 4 \AA$ (Fig. $3 \mathrm{~b}$ ), which is another previously observed difference between the holo and apo states of $\mathrm{Glt}_{\mathrm{Tk}}$ and $\mathrm{Glt}_{\mathrm{Ph}} 11,12,33$.

Substrate unsaturated conditions. Using a sub-saturating aspartate concentration, in which we aimed to occupy $\sim 1 / 3$ rd of the aspartate binding sites in the nanodisc preparation, we were able to solve two distinct structures, with different asymmetric arrangements of the transporter domains (Fig. 1). Roughly equal amounts of particles were used for the two reconstructions (Supplementary Fig. 1c). The individual protomers in the two structures are either in the inward-open state, identical to the state observed in the $\mathrm{Na}^{+}$only condition, or in the outward-intermediate state, identical to the ones observed under aspartate-saturated conditions. The difference between the two structures is the number of protomers in each state, with either two inward and one intermediate-outward (2 in:1 out), or one inward and two intermediate-outward (1 in:2 out) oriented protomers. Our data indicate that we visualized a part of 

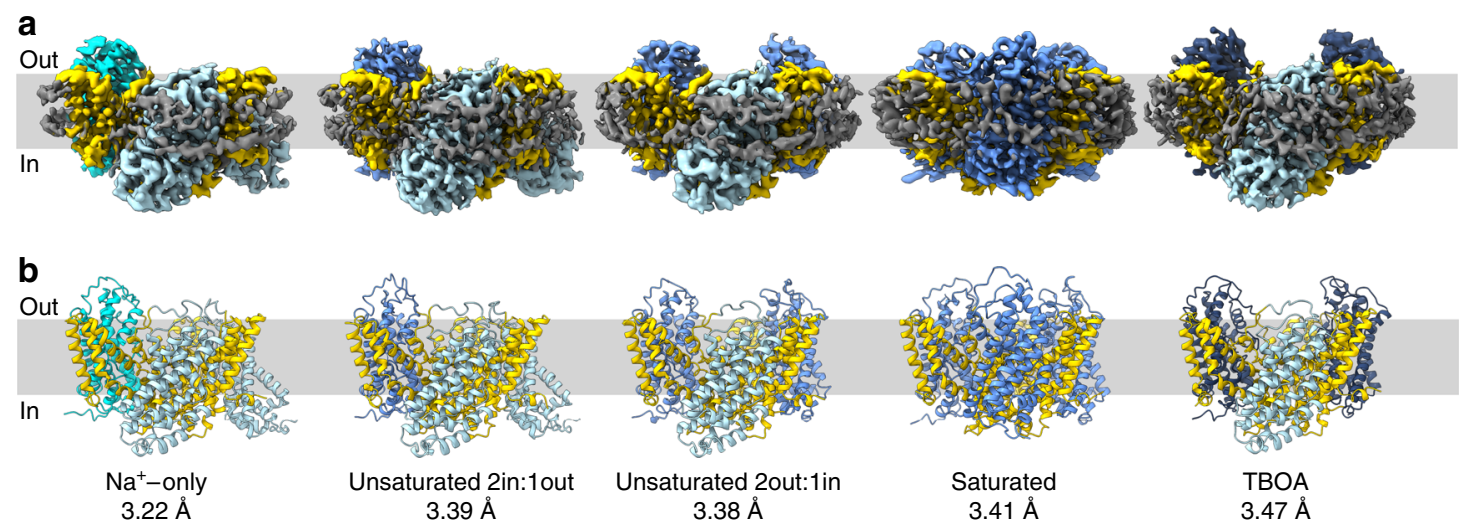

\begin{tabular}{|l|l|l|l|}
\hline Ctructure & \multicolumn{1}{|c|}{ A } & \multicolumn{1}{c|}{ B } & \multicolumn{1}{c|}{ C } \\
\hline $\mathrm{Na}^{+}$-only & $\begin{array}{l}\text { Intermediate-outward } \\
\text { occluded, apo }\end{array}$ & Inward open & Inward open \\
\hline Unsaturated 2in:1out & Inward open & Inward open & $\begin{array}{l}\text { Intermediate-outward } \\
\text { occluded, Asp }\end{array}$ \\
\hline Unsaturated 2out:1in & Inward open & $\begin{array}{l}\text { Intermediate-outward } \\
\text { occluded, Asp }\end{array}$ & $\begin{array}{l}\text { Intermediate-outward } \\
\text { occluded, Asp }\end{array}$ \\
\hline Saturated & $\begin{array}{l}\text { Intermediate-outward } \\
\text { occluded, Asp }\end{array}$ & $\begin{array}{l}\text { Intermediate-outward } \\
\text { occluded, Asp }\end{array}$ & $\begin{array}{l}\text { Intermediate-outward } \\
\text { occluded, Asp }\end{array}$ \\
\hline TBOA & Inward open & $\begin{array}{l}\text { Outward } \\
\text { open, TBOA }\end{array}$ & $\begin{array}{l}\text { Outward } \\
\text { open, TBOA }\end{array}$ \\
\hline
\end{tabular}

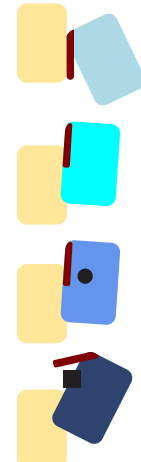

Fig. 1 Conformational states of the trimeric Glt $\mathbf{T}_{\mathbf{T k}} \mathbf{a}$, $\mathbf{b}$ Volume and cartoon representation of five cryo-EM structures of GIt $\mathrm{T}_{\mathrm{Tk}}$ in MSP2N2 nanodiscs obtained in the absence of substrate ( $\mathrm{Na}^{+}$-only), in presence of L-aspartate (unsaturated 2in:1out, unsaturated 1in:2out, saturated) or in the presence of DL-TBOA. The approximate position of the lipid bilayer is represented by the light gray bar, with indication of sides of the membrane (in and out). a The cryo-EM density for the MSP2N2 belt is shown in dark gray. The transport domains of the individual protomers are present in four different conformations: inward open (steel blue), intermediate-outward occluded apo (cyan), intermediate-outward occluded Asp (cornflower blue), outward-open TBOA (dark blue). The scaffold domains are shown in yellow. c Table summarizing conformations of Glt $t_{T k}$ protomers. Schematic representation of the conformations on the right in the same colors as in $\mathbf{a}, \mathbf{b}$, with indication of L-aspartate (black circle), DL-TBOA (black square) and HP2 (dark red stick).
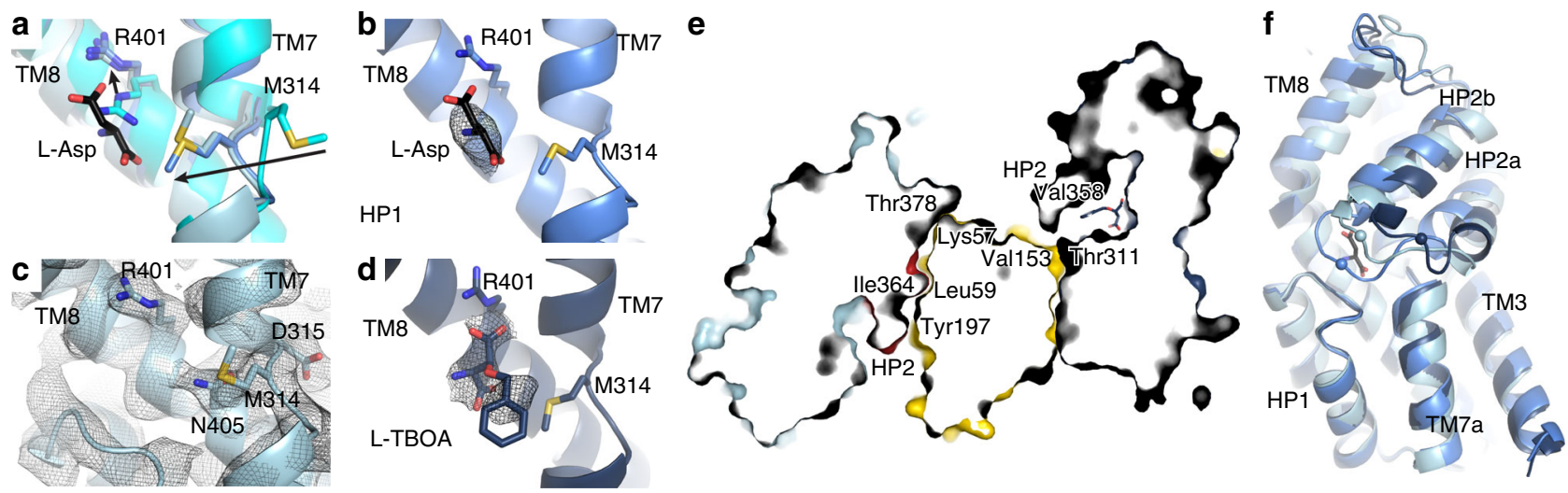

Fig. 2 Glt $\mathbf{t}_{\mathbf{T k}}$ substrate binding site and HP2 opening. a Overlay of apo (cyan), holo (Asp) (cornflower blue) and Na+-only states (light blue). The shift of Met314 and Arg401 is shown with arrows. b Cryo-EM density of L-aspartate (black mesh at $5 \sigma$ ). c Absence of the substrate in the inward open state (density is shown as gray mesh at $5 \sigma$ ). d Cryo-EM density of L-TBOA (black mesh at $4 \sigma$ ). e Slice through of TBOA-inhibited Glt $T_{T k}$ structure (surface representation) showing an inward-oriented and outward-oriented protomer. Opening of HP2 on both sides of the membrane prevents movements of the transport domains. $\mathbf{f}$ Superposition (on HP1) of the transport domains in inward $\mathrm{Na}^{+}$-only, intermediate-outward holo-Asp and fully-outward TBOAinhibited (dark blue) states. Opening of HP2 in the inward state (4.4 $\AA$ ) and TBOA-inhibited state $(10.4 \AA)$ in comparison with the occluded state is measured using $\mathrm{C} \alpha$ of $\mathrm{Val} 358$ (shown as sphere). L-aspartate (black sticks) indicates position of the substrate-binding site.

the multinomial distribution of the transport domains over all different possible states. The frequency of particles found with "2 in: 1 out" or " 1 in: 2 out" arrangement ( $89 \%$ of all particles) indicates that the inward-open and holo outward-intermediate states had the highest probabilities of occurrence under the substrate-unsaturated conditions. It is possible that more states and a more complete multinomial distribution could be resolved if many more particles were collected. 
a

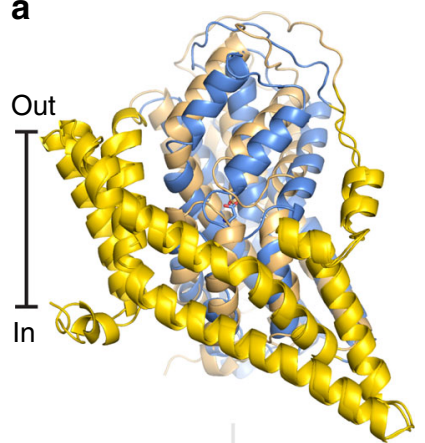

$70^{\circ}$

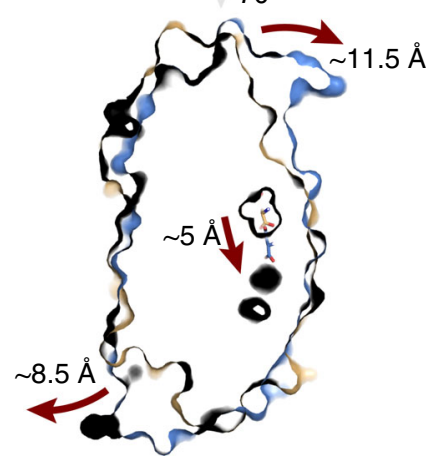

b
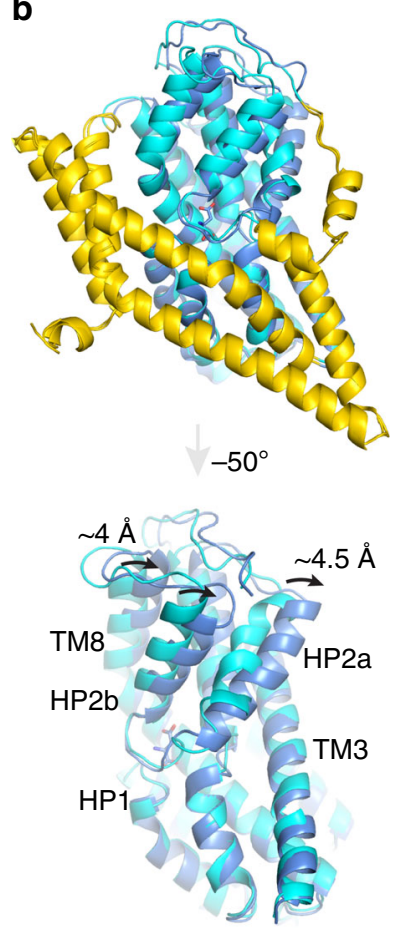

C

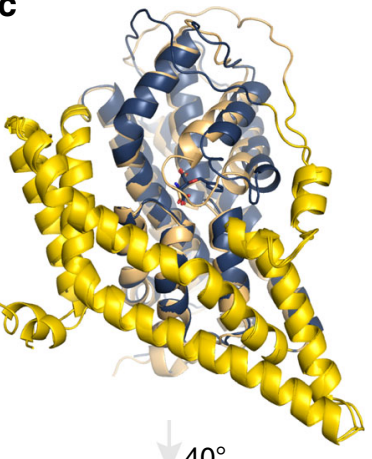

$40^{\circ}$

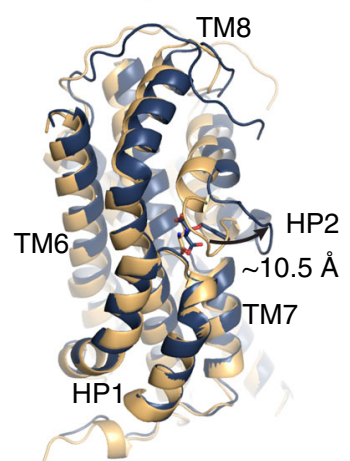

d
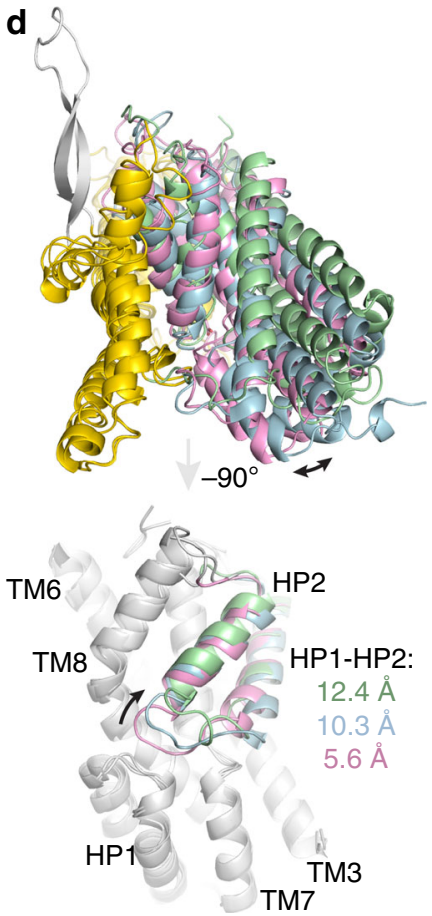

Fig. 3 Conformational differences of the Glt $\mathbf{t}_{\mathbf{T k}}$ protomers. a Comparison of the holo (Asp) Glt $\mathrm{T}_{\mathrm{Tk}}$ cryo-EM (cornflower blue) and crystal (PDB 5E9S, light orange $)^{12}$ structures (superposition using scaffold domains (yellow)) demonstrates differences between the fully outward and intermediate outward states. The panel below shows a slice through the transport domains in surface representation with aspartate molecules shown as sticks. The arrows indicate the movement of the transport domain from the fully-outward to intermediate-outward position. b Superposition on the scaffold domains of the cryo-EM structures of $\mathrm{Glt}_{\mathrm{Tk}^{-}}$holo (Asp) (cornflower blue) and $\mathrm{Glt}_{\mathrm{Tk}}$-apo (cyan), which are both in intermediate-outward conformations. The lower panel shows the transport domains rotated $50^{\circ}$ relative to the upper panel to highlight structural differences. c Superposition on the scaffold domains of cryo-EM structure of $\mathrm{Glt}_{\mathrm{Tk}}-\mathrm{TBOA}$ (dark blue) and crystal structure $\mathrm{Glt}_{\mathrm{Tk}}$-Asp (PDB 5E9S, light orange). The lower panel shows the transport domains rotated $40^{\circ}$ relative to the upper panel with an arrow indicating opening of the HP2 gate. d Superposition of the cryo-EM structure of $\mathrm{Glt}_{\mathrm{Tk}}-\mathrm{Na}{ }^{+}$-only (light blue), the cryo-EM structure of ASCT2 (PDB 6RVX, light green) ${ }^{17}$, and a crystal structure of Glt $\mathrm{Ph}_{\mathrm{p}}$-Asp in the inward-oriented state (PDB 3KBC, pink) ${ }^{10}$. The lower panel highlights opening of the HP2 gate in the inward-oriented state. Superpositions on TM2 and TM5 of the scaffold domain.

TBOA-inhibited condition. In the four structures presented above, none of the protomers was in a fully outward orientation, which is remarkable, because this orientation is most frequently observed in crystal structures of both $\mathrm{Glt}_{\mathrm{Ph}}$ and $\mathrm{Glt}_{\mathrm{Tk}}$. To test whether this state could be visited in our nanodisc preparations, we determined a structure in the presence of $120 \mu \mathrm{M}$ DL-TBOA. This bulky competitive inhibitor of aspartate transport sterically prevents the closure of the HP2 gate, and traps the transporter in an open state, which in the crystal structures of $\mathrm{Glt}_{\mathrm{Ph}}$ is exclusively fully-outward (PDB 2NWW) ${ }^{9}$. Cryo-EM analysis of $\mathrm{Glt}_{\mathrm{Tk}}$ in this condition shows an asymmetric trimer with one transport domain positioned in an inward-oriented state and two domains in a fullyoutward-state. The structure of the inward-oriented protomer is identical to the ones described above, and is similarly interpreted as representing a $\mathrm{Na}^{+}$-only state. The fully-outward protomers have wide open HP2 gates (distance between the tips of HP1 and HP2 13.8-14.5 $\AA$ ), and show density for bound TBOA (Figs. 2d, $3 \mathrm{c})$. The structures of these protomers are similar to those of crystal structures of $\mathrm{Glt}_{\mathrm{Ph}}$ in the presence of TBOA (rmsd 0.670 $\AA$ ), and show that the fully-outward conformation is accessible in the nanodisc environment. This structure also reveals that the tip of HP1 of Glt $\mathrm{Tk}_{\mathrm{k}}$ moves as much as $24 \AA$ across the lipid bilayer in the transition between inward and outward states (Supplementary Table 2). The asymmetric $\mathrm{Glt}_{\mathrm{Tk}}$ structure with only two protomers occupied by the inhibitor suggests that the concentration of TBOA used was not saturating, which is consistent with the concentrations of $\mathrm{Na}^{+}$, DL-TBOA and protein used in the experiment, expected to lead to $\sim 83 \%$ saturation.
Lipids and membrane scaffold protein. We selected the MSP2N2 belt protein for reconstitution, because it is able to form nanodiscs with large diameter $(15 \mathrm{~nm})^{37}$, however our structures show that their diameter is only $\sim 10-11 \mathrm{~nm}$, and the belt wraps around the protein more tightly (Supplementary Fig. 5). Nonetheless, we deduce from the structures that the transport domains had the freedom to move between inward and outward positions while embedded in the nanodiscs. All nanodisc samples were prepared in an identical way in the absence of substrates. The observation that redistributions of the transport domains to different conformations occurred upon addition of L-aspartate or DL-TBOA to the Glt $_{\mathrm{Tk}}$ nanodiscs, shows that the belt protein did not prevent movement of the transport domains across the membrane.

Cryo-EM densities resembling phospholipids in a bilayer-like arrangement were found in the crevices between the protomers of the Glt $_{\mathrm{Tk}}$ trimers, and indicate the position of the membrane (Fig. 4a). Since lipid-like densities were found only in these crevices, it was not possible to follow the shape of the lipid bilayer along the entire perimeter of the reconstituted protein. Therefore, we inspected the conformation of the MSP2N2 belt proteins, which were resolved in all structures obtained here. We used them as a guide to locate the position of the bilayer, also in places where lipid densities were not visible, and to estimate the extent of membrane deformations. We oriented the $\mathrm{Glt}_{\mathrm{Tk}}$ trimers with the $z$-axis along the pseudo three-fold axis of the scaffold domains, and then plotted the $y$-coordinates of the atoms from the modeled MSP2N2 protein as a function of the perimeter 


\section{a}
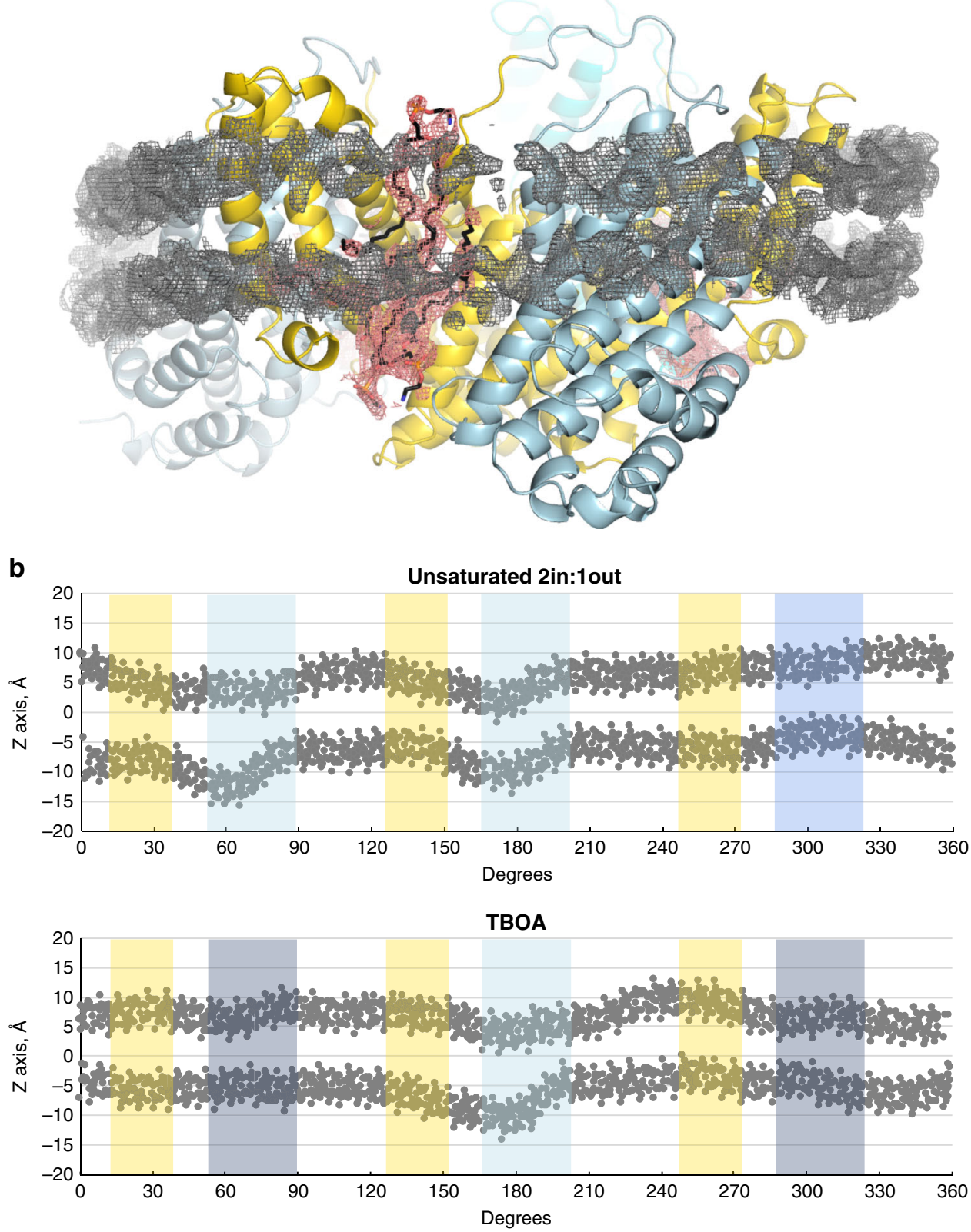

Fig. 4 Lipids and membrane deformation. a MSP2N2 (gray mesh, $3.5 \sigma$ ) and lipids (black sticks, salmon mesh, $3 \sigma$ ) in the Glt $t_{T k}$ structure determined in $\mathrm{Na}+$-only conditions. Colors are the same as in Figs. 1-3. b Glt $t_{T k}$ trimers were oriented with the $z$-axis along the pseudo three-fold axis of the scaffold domains, and the $y$-coordinates of the atoms from the modeled MSP2N2 protein were plotted as a function of the position along the $360^{\circ}$ perimeter. The vertical colored bars indicate where the belt covers the scaffold (yellow) and transport domains (shades of blue as in Fig. 1c). Source data are provided as a Source Data file.

position (Fig. 4b). A straight horizontal conformation of the belt protein indicates a planar lipid bilayer, whereas buckling of the belt protein is indicative of membrane deformation. The straightest conformation of the MSP2N2 protein was found around the protomers with outward oriented transport domains, whereas the largest extent of buckling was found around the inward-oriented protomers. Apparently, buckling of the belt proteins did not lead to strongly disfavored conformations of $\mathrm{Glt}_{\mathrm{Tk}}$ in the nanodiscs, as in 6 out of the 15 protomers in the collective set of structures the transport domains are in inward positions. A recent molecular dynamics simulation on $\mathrm{Glt}_{\mathrm{Ph}}$ also showed perturbations of the lipid bilayer around inward-oriented transport domains of $\mathrm{Glt}_{\mathrm{Ph}}{ }^{38}$. The buckling of the belt proteins in our structures resembles these perturbations, indicating that the conformation of belt proteins provides at least an approximate position of the bilayer.

\section{Discussion}

The collection of cryo-EM structures of nanodisc-reconstituted $\mathrm{Glt}_{\mathrm{Tk}}$ presented here, reveals a variety of asymmetric protomer arrangements in the trimeric protein, consistent with a transport mechanism in which the protomers operate independently from each other, as shown by smFRET ${ }^{21,22}, \mathrm{AFM}^{23}, \mathrm{MD}$ simulations ${ }^{24}$, and mutagenesis studies ${ }^{18}$. The individual protomers were found in four different states, and cover the full trajectory between inward-open and outward-open states. Two of the elucidated 
states have not been observed previously: a $\mathrm{Na}^{+}$-only inwardopen state, and an apo-occluded intermediate-outward state.

The $\mathrm{Na}^{+}$-only state provides insight in the mechanism by which substrate and $\mathrm{Na}^{+}$leakage is prevented, and thus strict coupling between $\mathrm{Na}^{+}$and aspartate transport is maintained. Leakage would occur if reorientation of the inward-open to the outward-open state were possible in the $\mathrm{Na}^{+}$-only state. The structure of the inward-open state presented here shows how this transition is prevented. The HP2 gate is open, and blocks movement of the transport domain. The same HP2 gate also opens in the outward-oriented state, captured here in the presence of TBOA, and prevents the transport domain from moving inward (Fig. 2e). In both cases, the tip and N-terminal helix of HP2 (HP2a) are displaced away from the aspartate binding site, and obstruct the movement of the transport domain along the scaffold (Fig. 2f). This mechanism, in which a single gate is used on both sides of the membrane resembles the one-gate elevator mechanism recently described for the neutral amino acid transporter ASCT2 ${ }^{17}$. The extent of gate opening in inward-open $\mathrm{Na}^{+}$only $\mathrm{Glt}_{\mathrm{Tk}}(\sim 9.3 \AA$ distance between the tips of HP1 and HP2) differs from the opening in inward-open ASCT2, but is large enough for substrates to pass. Also, the position of the inwardoriented transport domains deviates somewhat between the structurally characterized proteins (Fig. 3d), which could be related to differences between detergent-solubilized and nanodisc-reconstituted proteins.

We had not anticipated to capture an inward-open state in the presence of only $\mathrm{Na}^{+}$, because for $\mathrm{Glt}_{\mathrm{Ph}}$ it has been shown that the outward-facing state binds $\mathrm{Na}^{+}$with three-fold higher affinity than the inward facing state ${ }^{34}$, and therefore the outward $\mathrm{Na}^{+}$only state was expected to be favored. Similarly, in a high-speed AFM study of Glt $_{\mathrm{Ph}}$ the outward facing state was favored in the presence of $1 \mathrm{M} \mathrm{Na}^{+23}$. The fact that $\mathrm{Glt}_{\mathrm{Tk}}$ was stable in the inward-open $\mathrm{Na}^{+}$-only state could be related to differences between detergent-solubilized protein (used for the Glt $t_{\mathrm{Ph}}$ binding assays), bilayers with densely packed, reconstituted Glt $_{\mathrm{Ph}}$ on a mica support (used for the AFM study), and nanodisc reconstituted protein (used for $\mathrm{Glt}_{\mathrm{Tk}}$ here), or bona fide differences between the two proteins. In living cells, the captured inwardopen conformation of $\mathrm{Glt}_{\mathrm{Tk}}$ would correspond to a state from which aspartate has been released following transport, and $\mathrm{Na}^{+}$ ions are on the verge of being released. It is unlikely to be a low energy state in vivo, because the intracellular $\mathrm{Na}^{+}$concentration is low, and therefore dissociation of $\mathrm{Na}^{+}$yielding the apo state will be favored. In the apo-state the HP2 gate can close, leading subsequently to reorientation of the transport domain to the intermediate-outward position, observed in apo structure presented here.

An intermediate-outward state is found in 7 of the 15 protomers from the five structures determined here, indicating that it is a low-energy conformation. A similar state was observed only for one protomer (Supplementary Fig. 3) in 34 available structures of $\mathrm{Glt}_{\mathrm{Ph}}{ }^{8-10,29,33,34,39-41}, \mathrm{Glt}_{\mathrm{Tk}}{ }^{11,12,28}$, EAAT1 ${ }^{26}$, and ASCT2 ${ }^{17,27,42}$. The prominent presence of the intermediate outward state in our cryo-EM structures suggests that it is favored in the membraneenvironment, whereas detergent solution or crystal packing may favor the fully outward state. The outward-intermediate position of the transport domain was observed both in the fully loaded holo-(Asp)-state, and in the apo-state, suggesting that it is used both in the substrate-transport step and in the return of the empty carrier (Supplementary Fig. 6). It is not clear whether the binding site can become accessible by opening of the HP2 gate when the transport domain is in the intermediate-outward state, or that a transition to the fully outward state is needed for gate opening. The structures in the presence of TBOA suggest the latter. In vivo, the apo-intermediate state may not be long-lived, as excursions to the outward-open state in the presence of high external $\mathrm{Na}^{+}$concentrations may stabilize a $\mathrm{Na}^{+}$-only state. We have been unable to capture the outward-open $\mathrm{Na}^{+}$-only state, because we could not introduce sidedness to the $\mathrm{Na}^{+}$addition, and the inward-open $\mathrm{Na}^{+}$-only state of $\mathrm{Glt}_{\mathrm{Tk}}$ in lipid nanodiscs is more stable at high $\mathrm{Na}^{+}$-concentration.

Despite the observation that $\mathrm{Glt}_{\mathrm{Tk}}$ is tightly wrapped by and can potentially interact with the belt proteins, it can still bind the substrates and undergo large conformational changes. Nevertheless, the close proximity of the belt protein and $\mathrm{Glt}_{\mathrm{Tk}}$ might affect the conformational ensemble and create artefacts similar to crystal contacts in protein crystals, or sub-optimal lipid compositions in bilayers ${ }^{43}$. Despite an increasing amount of cryo-EM structures of membrane proteins in nanodiscs, discussions on the tightness of the belt protein are very often omitted.

In conclusion, the collection of structures presented here provides insight into the conformational ensemble of a secondary active transporter in conditions where conformational changes related to transport take place, similar to what has been done for ATP-driven transporters (the $\mathrm{ABC}$ transporter $\mathrm{TmrAB}^{44}$ and the mitochondrial ATP synthase ${ }^{45}$ ). Our work reveals a rich diversity of previously undetected asymmetric states of $\mathrm{Glt}_{\mathrm{Tk}}$, consistent with independent activity of the protomers. At the same time, our analysis shows that a lack of compartments, cation gradients and membrane voltage may obscure mechanistic details. Future developments in structural analysis of liposome-reconstituted proteins may open the way for structure determination under transporting conditions ${ }^{46}$.

\section{Methods}

Production and purification of MSP2N2. The gene encoding the membrane scaffold protein MSP2N2 with the $\mathrm{His}_{7}$-tag at the N-terminus cloned in the pET28a plasmid (Addgene) was expressed in E.coli BL21(DE3) cells (New England Biolabs). Cells were cultivated in LB medium in the presence of $50 \mu \mathrm{g} / \mathrm{mL}$ of kanamycin at $37^{\circ} \mathrm{C}, 200$ r.p.m. until the $\mathrm{OD}_{600}$ reached 0.8 . Then expression was induced by addition of $0.1 \mathrm{mM}$ IPTG and after $3.5 \mathrm{~h}$ cells were harvested by centrifugation $\left(15 \mathrm{~min}, 6270 \times \mathrm{g}, 4^{\circ} \mathrm{C}\right)$. Cells were resuspended in $40 \mathrm{mM}$ Tris- $\mathrm{HCl}$ $\mathrm{pH} 7.8,300 \mathrm{mM} \mathrm{NaCl}$ and stored at $-80^{\circ} \mathrm{C}$. For purification cells were thawed and supplemented with $100 \mu \mathrm{g} / \mathrm{mL}$ DNase A, $1 \mathrm{mM} \mathrm{MgSO}_{4}$ and $1 \mathrm{mM}$ PMSF. Cells were passed through a cell disrupter at $4{ }^{\circ} \mathrm{C}$ and $25 \mathrm{kPsi}$ (Constant Systems Ltd. Daventry UK). One percent of TritonX-100 was added to the lysate followed by stirring for $10 \mathrm{~min}$ at room temperature. After centrifugation at $30,000 \times \mathrm{g}, 30 \mathrm{~min}$, $4^{\circ} \mathrm{C}$ supernatant was supplemented with $20 \mathrm{mM}$ Imidazole, $\mathrm{pH}$ 8.0, and MSP2N2 was purified using Ni-NTA resin. MSP2N2 was eluted in $40 \mathrm{mM}$ Tris- $\mathrm{HCl}$, $\mathrm{pH} 8.0$, $300 \mathrm{mM} \mathrm{NaCl}, 500 \mathrm{mM}$ Imidazole. To cleave off the His-tag, protein fractions were pooled, supplemented with $1 \mathrm{mM} \mathrm{Na-EDTA}$ and 1:40 w/w TEV (Sigma-Aldrich) and dialyzed against $20 \mathrm{mM}$ Tris- $\mathrm{HCl}, \mathrm{pH} 8.0,100 \mathrm{mM} \mathrm{NaCl}, 0.5 \mathrm{mM}$ EDTA, 0.5 $\mathrm{mM}$ DTT overnight at $4{ }^{\circ} \mathrm{C}$ using a Servapor ${ }^{\circledast}$ dialysis tubing. The cleaved protein was loaded onto Ni-NTA resin and collected in the flow-through fraction using 50 $\mathrm{mM}$ Tris- $\mathrm{HCl}, \mathrm{pH} 8.0,100 \mathrm{mM} \mathrm{NaCl}$. Protein fractions were stored at $-80^{\circ} \mathrm{C}$.

Production and purification of apo $\mathbf{G l t}_{\mathbf{T k}}$. For production of C-terminally $\mathrm{His}_{8}$ tagged $\mathrm{Glt}_{\mathrm{Tk}}$, E. coli MC1061 containing a pBAD24 derived plasmid were grown in $\mathrm{LB}$ medium with $100 \mu \mathrm{g} / \mathrm{mL}$ ampicillin at $37^{\circ} \mathrm{C}, 200$ r.p.m. When the $\mathrm{OD}_{600}$ reached 0.8 expression was induced with $0.05 \% \mathrm{~L}$-arabinose for $3 \mathrm{~h}$. Cells were harvested by centrifugation $\left(15 \mathrm{~min}, 7400 \times g, 4^{\circ} \mathrm{C}\right.$ ) and resuspended in $20 \mathrm{mM}$ Tris- $\mathrm{HCl}, \mathrm{pH}$ 8.0. After breaking cells $\left(25 \mathrm{kPsi}, 5^{\circ} \mathrm{C}\right.$, Constant Systems Ltd. Daventry UK) the membrane fraction was collected by ultracentrifugation of the supernatant $\left(90 \mathrm{~min}, 193,360 \times g, 4^{\circ} \mathrm{C}\right)$, resuspended in $20 \mathrm{mM}$ Tris- $\mathrm{HCl}, \mathrm{pH} 8.0$, and stored at $-80^{\circ} \mathrm{C}$. To obtain apo-Glt ${ }_{\mathrm{Tk}} \mathrm{Na}^{+}$was omitted from all buffers. An aliquot of membrane vesicles representing $\sim 1.2 \mathrm{~g}$ cells was solubilized in $50 \mathrm{mM}$ Tris- $\mathrm{HCl}, \mathrm{pH} 8.0,300 \mathrm{mM} \mathrm{KCl}, 1 \% \mathrm{n}$-dodecyl- $\beta$-D-maltoside (DDM) for $1 \mathrm{~h}$ at $4{ }^{\circ} \mathrm{C}$. After ultracentrifugation $\left(30 \mathrm{~min}, 265,000 \times g, 4^{\circ} \mathrm{C}\right)$ the supernatant was incubated with Ni-Sepharose resin (GE Healthcare) for $1 \mathrm{~h}$ at $4{ }^{\circ} \mathrm{C}$. The column was washed with $50 \mathrm{mM}$ Tris $\mathrm{HCl}, \mathrm{pH} 8.0,300 \mathrm{mM} \mathrm{KCl}, 0.15 \% \mathrm{n}$-decyl- $\beta$-Dmaltoside (DM), $60 \mathrm{mM}$ Imidazole, $\mathrm{pH} 8.0$, and the protein was eluted with the same buffer containing $500 \mathrm{mM}$ Imidazole. $\mathrm{Glt}_{\mathrm{Tk}}$ was further purified by size exclusion chromatography on a Superdex 200 10/300 gel-filtration column (GE Healthcare) in $10 \mathrm{mM}$ Hepes KOH, pH 8.0, $100 \mathrm{mM} \mathrm{KCl,} \mathrm{0.15 \%} \mathrm{DM.}$

Nanodiscs reconstitution and cryo-EM sample preparation. Twenty milligram per milliliter of liposomes containing E. coli polar lipids and egg PC (w/w 3:1, Avanti), were solubilized by adding $30 \mathrm{mM}$ DDM followed by $1 \mathrm{~min}$ vortexing and 
$3 \mathrm{~h}$ incubation at $4{ }^{\circ} \mathrm{C}$ while nutating. Freshly prepared apo $\mathrm{Glt}_{\mathrm{Tk}}$, cleaved MSP2N2 and solubilized lipids were mixed in 3:5:100 molar ratio (considering a single protein chain) and incubated at $4{ }^{\circ} \mathrm{C}$ for $90 \mathrm{~min}$ while nutating. To remove the detergent BioBeads (Bio-Rad) were added and the mixture was incubated overnight at $4{ }^{\circ} \mathrm{C}$. After removing BioBeads using a syringe the solution was supplied with 15 $\mathrm{mM}$ Imidazole, $\mathrm{pH} 8.0$, and Ni-NTA resin, equilibrated with $50 \mathrm{mM}$ Tris- $\mathrm{HCl}, \mathrm{pH}$ $8.0,100 \mathrm{mM} \mathrm{KCl}$ to remove empty nanodiscs. After $1 \mathrm{~h}$ incubation at $4{ }^{\circ} \mathrm{C}$ on a rocking platform resin was washed with $50 \mathrm{mM}$ Tris- $\mathrm{HCl}, \mathrm{pH} 8.0,300 \mathrm{mM} \mathrm{KCl}$ $30 \mathrm{mM}$ Imidazole, $\mathrm{pH} 8.0$ and nanodiscs were eluted with $500 \mathrm{mM}$ Imidazole in the same buffer. The sample was centrifuged $\left(10 \mathrm{~min}, 20,000 \times g, 4^{\circ} \mathrm{C}\right)$ and applied to Superdex 200 10/300 gel-filtration column (GE Healthcare) equilibrated with $20 \mathrm{mM}$ Tris- $\mathrm{HCl}, \mathrm{pH} 8.0,100 \mathrm{mM} \mathrm{NaCl}$. Fractions with nanodiscs were concentrated using VivaSpin ${ }^{\circledR} 500$ MWCO $50 \mathrm{kDa}$ concentrators (Sartorius) to 1-4 $\mathrm{mg} / \mathrm{mL}$. The sample was supplemented with $\mathrm{NaCl}$ to $300 \mathrm{mM}$ ( $\mathrm{Na}^{+}$-only state) and additionally with $\mathrm{L}$-aspartate (Sigma-Aldrich) at the molar ratios $\mathrm{Glt}_{\mathrm{Tk}}$ : $\mathrm{L}$-aspartate equal to 3:1 for unsaturated state ( $9 \mu \mathrm{M}$ of both nanodiscs and L-aspartate) or 1:3 for saturated state $(5.6 \mu \mathrm{M}$ nanodiscs and $50 \mu \mathrm{M}$ L-aspartate) or with DL-TBOA $(4.5 \mu \mathrm{M}$ nanodiscs and $120 \mu \mathrm{M}$ DL-TBOA). After mixing the sample was incubated for $30 \mathrm{~min}$ on ice. The sample at $0.5-1 \mathrm{mg} / \mathrm{mL}$ was applied onto freshly glowdischarged Quantifoil grids (Au R1.2/1.3, 300 mesh) at $22^{\circ} \mathrm{C}$ and $100 \%$ humidity and plunged-frozen in liquid ethane. The Cryo-EM data were collected using 200 keV Talos Arctica microscope (Thermo Fisher).

Cryo-EM image processing. Motion correction, CTF estimation, template-based picking, 2D classification, $\mathrm{Ab}$ initio volume generation and non-uniform $3 \mathrm{D}$ refinement (without symmetry applied if not stated otherwise) were performed using cryoSPARC ${ }^{47}$ (Supplementary Fig. 1). Maps were sharpened using Autosharpen Map procedure in Phenix ${ }^{48}$. The sharpened maps were used for the manual model building using $\operatorname{Coot}^{49}$ and refinement of the coordinates was performed in realspace refine module of Phenix ${ }^{50,51}$. Visualization and structure interpretation were carried out in UCSF Chimera ${ }^{52,53}$ and PyMol

(Schrödinger, LLC).

For the data obtained using protein in aspartate-free conditions, 1288 micrographs were selected for the processing after motion correction and CTF estimation. The template for particle picking was generated from 100 manually picked particles. Template-based picking identified 1,193,046 particles. Subsequent 2D classification reduced the number of particles to 334,624 and subsequently $180,608(49 \%)$ particles were left in the selected ab initio class. Final non-uniform $3 \mathrm{D}$ refinement resulted in a $3.22 \AA$ map, where one protomer was oriented up (apo state) and two protomers were inward oriented. Other particles from ab initio volume generation were combined and refined, providing a low-resolution map of $4.82 \AA$ ( 2 out: 1 in).

For the data obtained using protein in substrate unsaturated conditions, 956 micrographs were selected after motion correction and CTF estimation. 348,173 particles were picked and 149,715 particles were left after subsequent 2D classification. Ab initio volume generation provided three classes of 72,313 (48\%), $59,666(40 \%)$, and 17,736 (12\%) particles. Two big classes were used for nonuniform 3D refinement without applying a symmetry and resulted in maps of $3.38 \AA$ ( 2 out: 1 in) and $3.39 \AA$ ( 2 in: 1 out).

For the data obtained using protein in substrate saturated conditions, 541 micrographs were selected after motion correction and CTF estimation. 444,955 particles were picked and 92,538 particles were left after subsequent 2D classification. Ab initio volume generation provided with one class of 65,762 (71\%) particles that was used for non-uniform 3D refinement without applying a symmetry. The resulting map of $4.0 \AA$ resolution revealed symmetrical arrangement of the three protomers, therefore $\mathrm{C} 3$ symmetry was applied to generate a final $3.41 \AA$ map.

For the data obtained using protein in TBOA-inhibited conditions, 1,675 micrographs were selected after motion correction and CTF estimation and 578,728 particles were picked. Following 2D classification a set of 228,131 particles was left. After ab initio volume generation one class of 132,917 (58\%) particles was chosen for further non-uniform $3 \mathrm{D}$ refinement without applying a symmetry. The resulting map of $3.47 \AA$ resolution revealed an asymmetric state with two fullyoutward and one inward oriented protomers.

Reporting summary. Further information on research design is available in the Nature Research Reporting Summary linked to this article.

\section{Data availability}

Data supporting the findings of this manuscript are available from the corresponding authors upon reasonable request. A reporting summary for this Article is available as a Supplementary Information file. The source data underlying Fig. $4 \mathrm{~b}$ is provided as a Source Data file. The cryo-EM density maps of the glutamate transporter homolog $\mathrm{Glt}_{\mathrm{Tk}}$ in $\mathrm{Na}^{+}$-only, unsaturated (2in:1 out), unsaturated (2out:1in), saturated conditions and in the presence of TBOA inhibitor have been deposited in the Electron Microscopy Data Bank under accession numbers EMD-10636, EMD-10633, EMD-10634, EMD-10635, EMD-10632, respectively. Coordinates of the corresponding five models have been deposited in the Protein Data Bank under the accession numbers 6XWR, 6XWO, 6XWP, 6XWQ and 6XWN, respectively.
Received: 13 January 2020; Accepted: 4 February 2020; Published online: 21 February 2020

\section{References}

1. Kanai, Y. \& Hediger, M. A. The glutamate/neutral amino acid transporter family SLC1: molecular, physiological and pharmacological aspects. Pflug. Arch. Eur. J. Physiol. 447, 469-479 (2004).

2. Kanner, B. I. \& Sharon, I. Active transport of L-glutamate by membrane vesicles isolated from rat brain. Biochemistry 17, 3949-3953 (1978).

3. Vandenberg, R. J. \& Ryan, R. M. Mechanisms of glutamate transport. Physiol. Rev. 93, 1621-1657 (2013).

4. Levy, L. M., Warr, O. \& Attwell, D. Stoichiometry of the glial glutamate transporter GLT-1 expressed inducibly in a Chinese hamster ovary cell line selected for low endogenous $\mathrm{Na}+$-dependent glutamate uptake. J. Neurosci. 18, 9620-9628 (1998).

5. Owe, S. G., Marcaggi, P. \& Attwell, D. The ionic stoichiometry of the GLAST glutamate transporter in salamander retinal glia. J. Physiol. 577, 591-599 (2006).

6. Zerangue, N. \& Kavanaugh, M. Flux coupling in a neuronal glutamate transporter. Nature 383, 634-637 (1996).

7. Slotboom, D. J., Konings, W. N. \& Lolkema, J. S. Structural features of the glutamate transporter family. Microbiol. Mol. Biol. Rev. 63, 293-307 (1999).

8. Yernool, D., Boudker, O., Jin, Y. \& Gouaux, E. Structure of a glutamate transporter homologue from Pyrococcus horikoshii. Nature 431, 811-818 (2004).

9. Boudker, O., Ryan, R. M., Yernool, D., Shimamoto, K. \& Gouaux, E. Coupling substrate and ion binding to extracellular gate of a sodium-dependent aspartate transporter. Nature 445, 387-393 (2007).

10. Reyes, N., Ginter, C. \& Boudker, O. Transport mechanism of a bacterial homologue of glutamate transporters. Nature 462, 880-885 (2009).

11. Jensen, S., Guskov, A., Rempel, S., Hänelt, I. \& Slotboom, D. J. Crystal structure of a substrate-free aspartate transporter. Nat. Struct. Mol. Biol. 20, 1224-1226 (2013).

12. Guskov, A., Jensen, S., Faustino, I., Marrink, S. J. \& Slotboom, D. J. Coupled binding mechanism of three sodium ions and aspartate in the glutamate transporter homologue GltTk. Nat. Commun. 7, 13420 (2016).

13. Arkhipova, V., Guskov, A. \& Slotboom, D. J. Analysis of the quality of crystallographic data and the limitations of structural models. J. Gen. Physiol. 149, 1091-1103 (2017).

14. Groeneveld, M. \& Slotboom, D. J. Na+: aspartate coupling stoichiometry in the glutamate transporter homologue GltPh. Biochemistry 49, 3511-3513 (2010).

15. Huang, Z. \& Tajkhorshid, E. Dynamics of the extracellular gate and ion-substrate coupling in the glutamate transporter. Biophys. J. 95, 2292-2300 (2008).

16. Shrivastava, I. H., Jiang, J., Amara, S. G. \& Bahar, I. Time-resolved mechanism of extracellular gate opening and substrate binding in a glutamate transporter. J. Biol. Chem. 283, 28680-28690 (2008).

17. Garaeva, A. A., Guskov, A., Slotboom, D. J. \& Paulino, C. A one-gate elevator mechanism for the human neutral amino acid transporter ASCT2. Nat. Commun. 10, 3427 (2019).

18. Grewer, C. et al. Individual subunits of the glutamate transporter EAAC1 homotrimer function independently of each other. Biochemistry 44, 11913-11923 (2005).

19. Leary, G. P., Stone, E. F., Holley, D. C. \& Kavanaugh, M. P. The glutamate and chloride permeation pathways are colocalized in individual neuronal glutamate transporter subunits. J. Neurosci. 27, 2938-2942 (2007).

20. Koch, H. P., Brown, R. L. \& Larsson, H. P. The glutamate-activated anion conductance in excitatory amino acid transporters is gated independently by the individual subunits. J. Neurosci. 27, 2943-2947 (2007).

21. Erkens, G. B., Hänelt, I., Goudsmits, J. M. H., Slotboom, D. J. \& van Oijen, A M. Unsynchronised subunit motion in single trimeric sodium-coupled aspartate transporters. Nature 502, 119-123 (2013).

22. Akyuz, N., Altman, R. B., Blanchard, S. C. \& Boudker, O. Transport dynamics in a glutamate transporter homologue. Nature 502, 114-118 (2013).

23. Ruan, Y. et al. Direct visualization of glutamate transporter elevator mechanism by high-speed AFM. Proc. Natl Acad. Sci. 114, 1584-1588 (2017).

24. Stolzenberg, S., Khelashvili, G. \& Weinstein, H. Structural intermediates in a model of the substrate translocation path of the bacterial glutamate transporter homologue GltPh. J. Phys. Chem. B 116, 5372-5383 (2012).

25. Jiang, J., Shrivastava, I. H., Watts, S. D., Bahar, I. \& Amara, S. G. Large collective motions regulate the functional properties of glutamate transporter trimers. Proc. Natl Acad. Sci. USA 108, 15141-15146 (2011).

26. Canul-Tec, J. C. et al. Structure and allosteric inhibition of excitatory amino acid transporter 1. Nature 544, 446-451 (2017).

27. Garaeva, A. A. et al. Cryo-EM structure of the human neutral amino acid transporter ASCT2. Nat. Struct. Mol. Biol. 25, 515-521 (2018). 
28. Arkhipova, V. et al. Binding and transport of D-aspartate by the glutamate transporter homolog GltTk. Elife 8, e45286 (2019).

29. Verdon, G. \& Boudker, O. Crystal structure of an asymmetric trimer of a bacterial glutamate transporter homolog. Nat. Struct. Mol. Biol. 19, 355-357 (2012).

30. Denisov, I. G. \& Sligar, S. G. Nanodiscs for structural and functional studies of membrane proteins. Nat. Struct. Mol. Biol. 23, 481-486 (2016).

31. Bayburt, T. H., Grinkova, Y. V. \& Sligar, S. G. Self-assembly of discoidal phospholipid bilayer nanoparticles with membrane scaffold proteins. Nano Lett. 2, 853-856 (2002).

32. Hoorens, M. W. H. et al. Glutamate transporter inhibitors with photocontrolled activity. Adv. Ther. 1, 1800028 (2018).

33. Verdon, G., Oh, S., Serio, R. N. \& Boudker, O. Coupled ion binding and structural transitions along the transport cycle of glutamate transporters. Elife 3, e02283 (2014)

34. Reyes, N., Oh, S. \& Boudker, O. Binding thermodynamics of a glutamate transporter homolog. Nat. Struct. Mol. Biol. 20, 634-640 (2013).

35. Bendahan, A., Armon, A., Madani, N., Kavanaugh, M. P. \& Kanner, B. I. Arginine 447 plays a pivotal role in substrate interactions in a neuronal glutamate transporter. J. Biol. Chem. 275, 37436-37442 (2000).

36. Hänelt, I., Jensen, S., Wunnicke, D. \& Slotboom, D. J. Low affinity and slow $\mathrm{Na}+$ binding precedes high affinity aspartate binding in the secondary-active transporter GltPh. J. Biol. Chem. 290, 15962-15972 (2015).

37. Grinkova, Y. V., Denisov, I. G. \& Sligar, S. G. Engineering extended membrane scaffold proteins for self-assembly of soluble nanoscale lipid bilayers. Protein Eng. Des. Sel. 23, 843-848 (2010).

38. Zhou, W. et al. Large-scale state-dependent membrane remodeling by a transporter protein. Elife 8, e50576 (2019).

39. Akyuz, N. et al. Transport domain unlocking sets the uptake rate of an aspartate transporter. Nature 518, 68-73 (2015).

40. Scopelliti, A. J., Font, J., Vandenberg, R. J., Boudker, O. \& Ryan, R. M. Structural characterisation reveals insights into substrate recognition by the glutamine transporter ASCT2/SLC1A5. Nat. Commun. 9, 38 (2018).

41. Oh, S. \& Boudker, O. Kinetic mechanism of coupled binding in sodiumaspartate symporter GltPh. Elife 7, e37291 (2018).

42. $\mathrm{Yu}, \mathrm{X}$. et al. Structural basis for the transport mechanism of the human glutamine transporter SLC1A5 (ASCT2). Elife 8, e48120 (2019).

43. McIlwain, B. C., Vandenberg, R. J. \& Ryan, R. M. Transport rates of a glutamate transporter homologue are influenced by the lipid bilayer. J. Biol. Chem. 290, 9780-9788 (2015).

44. Hofmann, S. et al. Conformation space of a heterodimeric ABC exporter under turnover conditions. Nature 571, 580-583 (2019).

45. Murphy, B. J. et al. Rotary substates of mitochondrial ATP synthase reveal the basis of flexible F1-Fo coupling. Science 364, eaaw9128 (2019).

46. Tonggu, L. \& Wang, L. Broken symmetry in the human BK channel Lige. (2018). https://doi.org/10.1101/494385.

47. Punjani, A., Rubinstein, J. L., Fleet, D. J. \& Brubaker, M. A. CryoSPARC Algorithms for rapid unsupervised cryo-EM structure determination. Nat. Methods 14, 290-296 (2017).

48. Terwilliger, T. C., Sobolev, O. V., Afonine, P. V. \& Adams, P. D. Automated map sharpening by maximization of detail and connectivity. Acta Crystallogr. Sect. D 74, 545-559 (2018).

49. Emsley, P., Lohkamp, B., Scott, W. G. \& Cowtan, K. Features and development of Coot. Acta Crystallogr. Sect. D 66, 486-501 (2010).

50. Afonine, P. V. et al. Real-space refinement in PHENIX for cryo-EM and crystallography. Acta Crystallogr. Sect. D 74, 531-544 (2018).
51. Afonine, P. V. et al. New tools for the analysis and validation of cryoEM maps and atomic models. Acta Crystallogr. Sect. D 74, 814-840 (2018).

52. Pettersen, E. F. et al. UCSF Chimera-a visualization system for exploratory research and analysis. J. Comput. Chem. 25, 1605-1612 (2004).

53. Goddard, T. D. et al. UCSF ChimeraX: meeting modern challenges in visualization and analysis. Protein Sci. 27, 14-25 (2018).

\section{Acknowledgements}

We thank J.S.H.C.U.Rheinberger for cryo EM sample preparation and data collection, $H$ R.Sikkema for help with initial data processing, C. Paulino for running an excellent cryoEM facility and helpful discussions, A.Garaeva and H.T.Postmus for help in expression of MSP2N2. The research was supported by NWO Vidi grant 723.014.002 to A.G. and NWO TOP grant 714.018 .003 to D.J.S.

\section{Author contributions}

Protein expression, purification, and sample preparation were performed by V.A. Model building and refinement were done by V.A. and A.G. D.J.S. and A.G. supervised the project at all stages. V.A., D.J.S., and A.G. wrote the manuscript.

\section{Competing interests}

The authors declare no competing interests.

\section{Additional information}

Supplementary information is available for this paper at https://doi.org/10.1038/s41467020-14834-8.

Correspondence and requests for materials should be addressed to A.G. or D.J.S.

Peer review information Nature Communications thanks the anonymous reviewer(s) for their contribution to the peer review of this work. Peer reviewer reports are available.

Reprints and permission information is available at http://www.nature.com/reprints

Publisher's note Springer Nature remains neutral with regard to jurisdictional claims in published maps and institutional affiliations.

Open Access This article is licensed under a Creative Commons Attribution 4.0 International License, which permits use, sharing, adaptation, distribution and reproduction in any medium or format, as long as you give appropriate credit to the original author(s) and the source, provide a link to the Creative Commons license, and indicate if changes were made. The images or other third party material in this article are included in the article's Creative Commons license, unless indicated otherwise in a credit line to the material. If material is not included in the article's Creative Commons license and your intended use is not permitted by statutory regulation or exceeds the permitted use, you will need to obtain permission directly from the copyright holder. To view a copy of this license, visit http://creativecommons.org/ licenses/by/4.0\%

(C) The Author(s) 2020 